\title{
STATUS OF THE CUORE EXPERIMENT
}

\section{Claudia Tomei*, for the CUORE Collaboration}

\author{
Sez. INFN di Roma, Roma I-00815, Italy \\ * corresponding author: claudia.tomei@roma1.infn.it
}

\begin{abstract}
The CUORE (Cryogenic Underground Observatory for Rare Events) experiment will search for neutrinoless double beta decay of ${ }^{130} \mathrm{Te}$, a rare nuclear process that, if observed, would demonstrate the Majorana nature of the neutrino and enable measurements of the effective Majorana mass. The CUORE setup consists of an array of 988 tellurium dioxide crystals, operated as bolometers, with a total mass of about $200 \mathrm{~kg}$ of ${ }^{130} \mathrm{Te}$. The experiment is under construction at the Gran Sasso National Laboratory in Italy. As a first step towards CUORE, the first tower (CUORE-0) has been assembled and will soon be in operation.
\end{abstract}

KEYWORDS: double beta decay, neutrino mass, majorana neutrinos, bolometers, cryogenic detectors.

\section{INTRODUCTION}

Neutrinoless double beta decay is a unique probe for addressing the open questions in neutrino physics: the nature of a neutrino (Dirac or Majorana), the absolute neutrino mass scale and the mass hierarchy. Neutrinoless double beta decay $(0 \vee \beta \beta)$ is an extremely rare nuclear process, never observed up to now ${ }^{1}$ It can take place through the exchange of a massive Majorana neutrino, and its occurrence violates conservation of the total lepton number, thus implying physics beyond the Standard Model (for a recent review see [1]).

The $0 \vee \beta \beta$ decay amplitude depends on the effective Majorana mass

$$
m_{\beta \beta}=\left|\sum_{k} U_{e k}^{2} m_{k}\right|,
$$

where $U_{e k}$ are the elements of the neutrino mixing matrix and $m_{k}$ are the neutrino mass eigenstates. The measurable quantity is the half-life, which is related to $m_{\beta \beta}$ by the formula

$$
\left(T_{1 / 2}^{0 \nu}\right)^{-1}=G_{0 \nu}(Q, Z)\left|M_{0 \nu}\right|^{2} \frac{\left|\left\langle m_{\beta \beta}\right\rangle\right|^{2}}{m_{e}^{2}},
$$

where $G^{0 \vee}$ is the phase-space factor and $M_{0 v}$ is the nuclear matrix element. From a measurement (or a lower limit in the case of a null result) on $T_{1 / 2}^{0 \nu}$ for any $\beta \beta$ emitting isotope, one can extract the value (or an upper limit) for the Majorana neutrino mass, assuming the knowledge of the phase space factor and the nuclear matrix element for the given isotope.

The phase space factors $G^{0 \vee}$ are known with good accuracy [3]. Unfortunately, the same is not true for the nuclear matrix elements $M_{0 v}$, which are extremely difficult to calculate and can only be estimated using approximations. The actual spread in $M_{0 v}$ calculation

\footnotetext{
${ }^{1}$ With the exception of a very controversial claim 2, which will be tested by current generation experiments.
}

for a given isotope is of a factor $\sim 2$, which is reflected in the uncertainty of the same entity on $m_{\beta \beta}$, for a given experimental result.

\section{1. $0 \nu \beta \beta$ IN EXPERIMENTS}

The signature of $0 \vee \beta \beta$ is the emission of two simultaneous electrons with summed energy equal to the $\mathrm{Q}$-value of the $\beta \beta$ emitter. For experiments able to measure the sum energy of the two electrons, the signal appears as a peak at the Q-value. Despite its simple signature, the detection of $0 \vee \beta \beta$ is extremely challenging due to the fact that the predicted half lives for this kind of decay are above $10^{25} \mathrm{y}$. The main experimental issues are the issues common to all experiments that search for rare events. The most crucial parameters are summarized in the formula for the experimental sensitivity,

$$
S^{0 \vee}=\ln 2 N_{\mathrm{A}} \cdot \frac{a \epsilon}{A}\left(\frac{M T}{B \Delta E}\right)^{1 / 2}
$$

where $N_{\mathrm{A}}$ is the Avogadro number, $a$ is the isotopic abundance, $\epsilon$ is the detection efficiency, and the other quantities have the usual meaning.

Many experiments, based on various isotopes, are presently dedicated to the search for $0 \vee \beta \beta$ decay. Some of them are already running, while others are in the construction phase and are expected to start data taking in the coming years. The first goal is to scrutinize the ${ }^{76} \mathrm{Ge}$ claim [2], corresponding to a half-life of $2.23 \pm 0.04 \times 10^{25} \mathrm{y}$ and, if confirmed, collect sufficient statistics for a precision measurement. If it is not confirmed, the next goal is to improve the half-life sensitivity above $10^{26} \mathrm{y}$, in order to enter the region of $m_{\beta \beta}$ allowed for the inverted hierarchy of neutrino masses.

The best experimental results (namely limits on the $0 \vee \beta \beta$ half-life for many isotopes) have been produced up to now with the use of calorimetric detectors (Heidelberg-Moscow 4, CUORICINO [5]), tracking 


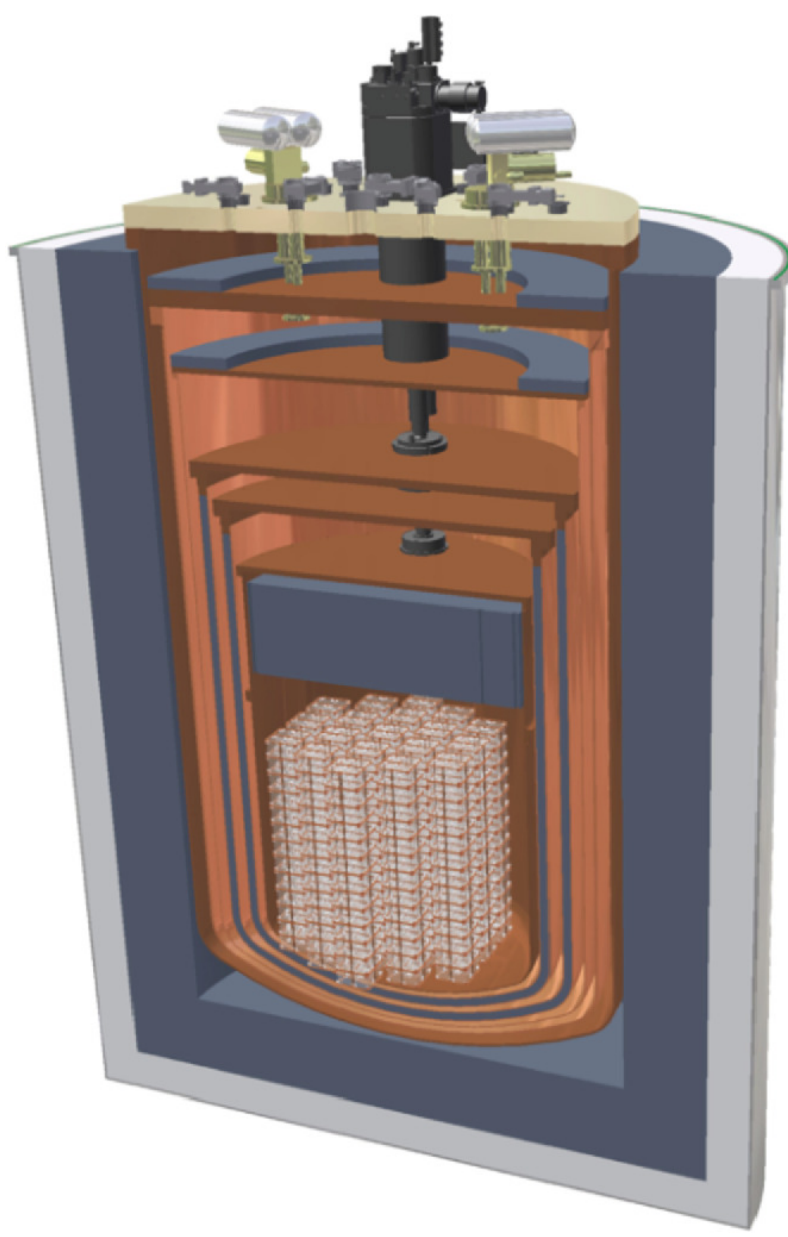

Figure 1. The CUORE experimental setup. A drawing of the full detector inside the future CUORE cryostat and shields.

detectors (NEMO-3 [6, 7]) and, more recently, liquid scintillator detectors (EXO [8, Kamland-Zen [9]).

\subsection{CUORE AND THE BOLOMETRIC TECHNIQUE}

Cryogenic bolometers are particle detectors where the energy of the impinging particle is converted into phonons, causing a temperature variation. Given the low heat capacity and the low base temperature $(\sim 10 \mathrm{mK})$ of the device, even small $\Delta T$ $(\sim 0.1 \mathrm{mK} / \mathrm{MeV})$ can be detected. The temperature rises are measured by thermistors glued to the surface of the bolometer.

CUORE bolometers [10, 11] are tellurium dioxide crystals. The choice of the absorber $\left(\mathrm{TeO}_{2}\right)$ is driven by many considerations: the excellent bolometric properties of this material, the high natural abundance of the $\beta \beta$ emitting isotope ${ }^{130} \mathrm{Te}(\sim 34 \%$, the highest among the $\beta \beta$ emitting isotopes used to date), the reasonably high Q-value $(2528 \mathrm{keV})$, almost above the energy spectrum of natural radioactivity, and the optimal energy resolution ( $\sim 5 \mathrm{keV} @$ the Q-value).

$\mathrm{TeO}_{2}$ crystals have been successfully operated for more than ten years in the search for the $0 \nu \beta \beta$ of ${ }^{130} \mathrm{Te}$. The best result obtained by the CUORICINO experi- ment is $T_{1 / 2}^{0 \vee}>2.8 \times 10^{24} \mathrm{y} @ 90 \%$ C.L. The future of the bolometric technique lies in the CUORE experiment, which will prove the feasibility of a $\sim 1$ ton scale experiment with cryogenic bolometers.

The CUORE setup consists in an array of 988 tellurium dioxide crystals for a total mass of about $200 \mathrm{~kg}$ of ${ }^{130} \mathrm{Te}$. The crystals will be arranged in 19 towers, each with 13 floors of 4 detectors, held by means of teflon spacers inside a copper structure. The whole array will be cooled down to $10 \mathrm{mK}$ in a cryostat, surrounded by a gamma and a neutron shield (see Fig. 11. The experiment is under construction at the Gran Sasso National Laboratory in Italy.

\subsection{CUORE BACKGROUND BUDGET}

The CUORE goal is to achieve a flat background in the region of interest (ROI) around the $0 \vee \beta \beta$ Q-value of 0.01 counts $/ \mathrm{kg} / \mathrm{keV} / \mathrm{y}$. This is quite a dramatic improvement for the CUORICINO background index $(0.169 \pm 0.006$ counts $/ \mathrm{kg} / \mathrm{keV} / \mathrm{y})$. For this purpose, only carefully selected radio-pure materials are used in the detector region, e.g. high-purity low-background copper and teflon. Moreover, the compact design and the granularity of the CUORE array will provide an additional background reduction through self-shielding and anti-coincidence between the detectors.

To shield against the external radioactivity, the detectors are surrounded by at least $30 \mathrm{~cm}$ of lead, divided into several layers. The innermost lead shields are made from ancient Roman lead with low contamination of ${ }^{210} \mathrm{~Pb}$. An additional $20 \mathrm{~cm}$ borated polyethylene layer surrounding the whole cryostat will act as a neutron shield.

The experience of CUORICINO has shown that an important fraction of the background in the ROI is due to degraded alpha from U/Th contaminations on the surfaces of crystals and of the mounting structure (copper and PTFE).

The CUORE crystals are purchased from SICCAS, and have been produced since 2009 following a very strict protocol to ensure the required radiopurity [12. Approximately $4 \%$ of the crystals delivered to LNGS are tested as cryogenic bolometers in the so-called CUORE Crystal Validation Runs (CCVRs). The results of the first five CCVRs have proven the excellent performance of the CUORE crystals in terms of energy resolution (improved with respect to CUORICINO) and the absence of U/Th surface and bulk contaminations 13 .

As far as the copper is concerned, all components facing the detectors have to undergo a complex cleaning process, following a procedure developed at the Legnaro National Laboratory of INFN. A small tower assembled using CUORICINO crystals (completely reprocessed according to the CUORE standard) was operated underground at LNGS, inside the CUORICINO cryostat, to measure the surface activity of the mounting structure 14. 


\begin{tabular}{|l|l|}
\hline Background Source & $\begin{array}{l}\text { B in ROI } \\
{[\text { counts } / \mathrm{kg} \mathrm{keV} \mathrm{y}]}\end{array}$ \\
\hline External $(\mu+\mathrm{n}+\gamma)$ & $<2 \times 10^{-3}$ \\
Roman lead (bulk) & $<4 \times 10^{-3}$ \\
Cosmogenic activation of crystals & $\sim 10^{-3}$ \\
Crystals (bulk) & $<1 \times 10^{-4}$ \\
Crystals (surface) & $<4 \times 10^{-3}$ \\
Mounting - Copper (surface) & $<(2 \div 3) \times 10^{-2}$ \\
Mounting - PTFE (surface) & $<6 \times 10^{-2}$ \\
\hline
\end{tabular}

TABle 1. Some contributions to CUORE background. The numbers and limits were obtained from bolometric tests, radioactivity measurements (HP-Ge, NAA) and Monte-Carlo simulations, and are reported in 13 15.

Some contributions to CUORE background are shown in Tab. 1. based on the pessimistic hypothesis that all the contributions observed in the R\&D tests come from sources that will not improve with the geometry of CUORE. The limits for the mounting structure are mutually exclusive.

\section{Status of the CUORE EXPERIMENT}

The CUORE experiment is presently under construction at the Gran Sasso National Laboratory (LNGS) of INFN. The CUORE experimental building (Hut) is located in Hall A of LNGS, and consists of three floors (cryostat equipment, clean rooms and electronics). The CUORE clean rooms are fully equipped for tower assembly. The assembly procedure is composed of several steps, each one taking place in a dedicated clean room.

The first phase of a the assembly of a CUORE tower is gluing the thermistors (temperature sensors) and the Joule heaters (silicon resistors to check fluctuations in the stability of the gain) to the crystals. This operation is performed within the thermistor gluing station, equipped with two robotic arms that ensure precise and uniform gluing.

The mechanical assembly of the tower is performed inside the assembly clean room, following the CUORE Tower Assembly Line (CTAL). The operational principle of CTAL is the following: a sealed and flushed stainless steel chamber (Garage) supports a common working plane (UWP), where 4 PMMA chambers (Glove Boxes) will switch, each one with features able to allow specific operations, namely mounting, bonding, cabling and storage, until completion of the tower. The CTAL procedure was successfully tested in May 2012 with the assembly of CUORE-0 (see below).

As concerns crystal production, more than $80 \%$ of the bolometers are already at LNGS, stored in nitrogen-fluxed cabinets in CUORE's underground Parts Storage Area (PSA).

The CUORE cryogenic system is a fundamental, though technologically challenging, part of the setup. It should ensure stable cooling of the detector's array to its operating temperature, while guaranteeing the operation of the detectors (readout and calibration) and proper shielding from environmental radiation. The key parts are the cryostat, the cooling unit and the fast cooling system, the readout (wiring), and the calibration systems.

The CUORE cryostat is made of six nested vessels at different temperatures, from $300 \mathrm{~K}$ (Outer Vacuum Chamber) to $0.01 \mathrm{~K}$ (Mixing Chamber). The cooling power to operate the CUORE detectors to temperatures in the $10 \mathrm{mK}$ range is provided by a cooling system based on a closed cycle, cryogen-free, high-power ${ }^{3} \mathrm{He} /{ }^{4} \mathrm{He}$ dilution refrigerator (DRS). The DRS was completed and successfully tested in July 2011. The cryostat commissioning at LNGS started in April 2012.

The calibration of the CUORE bolometers is also challenging. Due to the thick lead shields and the large self-shielding effect of the bolometer array, sources must be inserted into the detector region, adhering to the strict heat-load requirements of the cryostat. The CUORE detector calibration system (DCS) consists of 12 radioactive source strings that are able to move, under their own weight, through a set of guide tubes that route them from outside the cryostat at $300 \mathrm{~K}$ to their locations between the bolometers in the detector region inside the cryostat. The guide tubes are made of stainless steel, or high-purity low-background copper, depending on their position in the cryostat and the thermal requirements. The source strings are moved into the detector region for calibrations, and removed during regular data taking. The DCS is under construction at the University of Wisconsin.

The updated CUORE schedule foresees the first cooldown of the full detector by fall 2014 .

\section{CUORE-0}

CUORE-0 is a single CUORE tower, assembled from detector components manufactured, cleaned and stored following the stringent protocols defined for CUORE, with the aim of testing the performance of the new detector assembly line and detector structure. At the same time, given its sensitive mass (about $11 \mathrm{~kg}$ of ${ }^{130} \mathrm{Te}$ ) and provided the background can be kept at a level lower than CUORICINO, CUORE-0 can have a physics program of its own, surpassing CUORICINO in sensitivity reach, while CUORE is being assembled (see Fig. 2] [17, and demonstrating the potential for Dark matter and Axion detection with the help of a new software trigger able to reduce the threshold to a few $\mathrm{keV}[16$.

CUORE-0 operations began in September 2011, when the crystals were successfully instrumented with thermistors and heaters using the new semiautomated CUORE gluing system. During March 2012, a test assembly of all the new CUORE-0 copper parts, without the crystals, was successfully carried out. The mechanical assembly of the tower was started immediately afterwards, and was completed successfully in April 


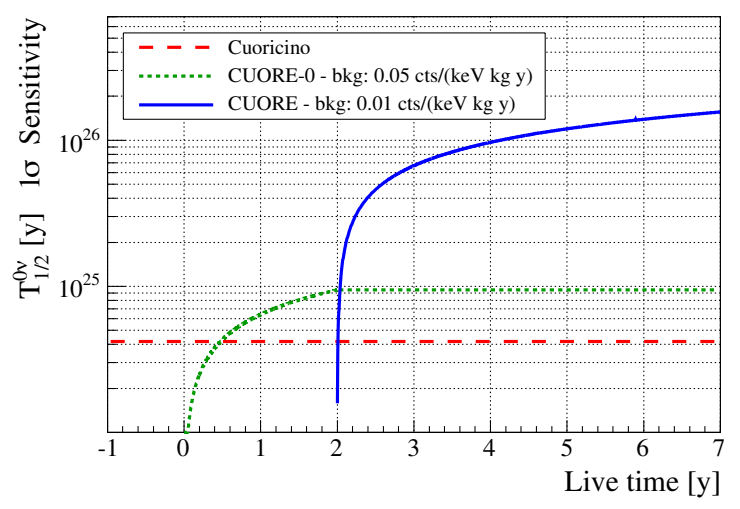

Figure 2. CUORE-0 sensitivity compared to the CUORICINO result and the CUORE expectations [17.

2012 with the installation of the copper shields. The fully assembled tower has been moved to the CUORICINO building for insertion into the cryostat and for cooldown. Unfortunately, a leak appeared in the dilution unit soon after the initial system check. The leak was then fixed, and the cooldown of CUORE-0 has started. Data taking was due to start at the end of August 2012.

\section{Conclusions}

The CUORE (Cryogenic Underground Observatory for Rare Events) experiment will search for neutrinoless double beta decay of ${ }^{130} \mathrm{Te}$. With a background of 0.01 counts $/ \mathrm{kg} / \mathrm{keV} /$ year and a lifetime of 5 years, CUORE will reach a sensitivity of $1.6 \times 10^{26} \mathrm{y} @ 68 \%$ C.L., thus entering for the first time the allowed region for the effective Majorana mass in the inverted hierarchy scenario. CUORE is under construction at the Gran Sasso National Laboratory in Italy, and cooldown of the detector is scheduled for fall 2014. As a first step towards CUORE, the first tower (CUORE-0) has been assembled and will soon come into operation.

\section{REFERENCES}

[1] S. Bilenky, C. Giunti: 2012, Mod.Phys.Lett. A27, 1230015 .

[2] H. V. Klapdor-Kleingrothaus, I. V. Krivosheina, A. Dietz, O. Chkvorets: 2004, Physics Letters B586, 198.

[3] J. Kotila and F. Iachello: 2012, Phys.Rev. C85, 034316.

[4] H. V. Klapdor-Kleingrothaus, et al.: 2001, Eur.Phys.J. $\mathrm{A} 12,147$.

[5] E. Andreotti, et al.: 2011, Astropart.Phys. 34, 822.

[6] R. Arnold, et al.: 2006, Nucl.Phys. A765, 483-494.

[7] A. Barabash, V. Brudanin: 2011, Phys.Atom.Nucl. 74, 312-317.

[8] M. Auger et al.: 2012, Phys.Rev.Lett. 109, 032505.

[9] KamLAND-Zen Collaboration: 2012, Phys.Rev.C 85, 045504.

[10] R. Ardito et al.: 2005, arXiv:hep-ex/0501010.

[11] C. Arnaboldi et al.: 2004, Nucl. Instr. Meth. A518, 775.

[12] C. Arnaboldi et al.: 2010, Journal of Crystal Growth $312,2999$.

[13] F. Alessandria et al.: 2012, Astropart.Phys. 35, 839-849.

[14] F. Alessandria et al.: 2013, Astropart.Phys. 45, 13-22.

[15] F. Bellini et al.: 2010, Astropart.Phys. 33, 169.

[16] S. Di Domizio, F. Orio and M. Vignati: 2011, JINST 6, P02007.

[17] F. Alessandria et al.: 2013, arXiv:1109.0494v3 [nucl-ex], submitted to Astropart.Phys.

\section{Discussion}

James Beall - What is the size of the CUORE crystals?

Claudia Tomei - Each crystal is $5 \times 5 \times 5 \mathrm{~cm}^{3}$. 